\title{
An Overview of Recent Nucleon Spin Structure Measurements at Jefferson Lab
}

\author{
Kalyan Allada*† \\ Massachusetts Institute of Technology \\ E-mail: kalyanejlab.org
}

\begin{abstract}
Our understanding of nucleon spin structure is still far from complete. Experiments conducted at Jefferson Lab have made significant contributions to improve our knowledge of the longitudinal spin structure by measuring polarized structure functions, $g_{1}$ and $g_{2}$, down to $\mathrm{Q}^{2}=0.02 \mathrm{GeV}^{2}$. The low $\mathrm{Q}^{2}$ data is especially useful in testing the Chiral Perturbation theory $(\chi \mathrm{PT})$ calculations. The spin-dependent sum rules and the spin polarizabilities, constructed from the moments of $g_{1}$ and $g_{2}$, provide an important tool to study the longitudinal spin structure. We will present an overview of the experimental program to measure these structure functions at Jefferson Lab, and present some recent results on the neutron polarizabilities, proton $g_{1}$ at low $\mathrm{Q}^{2}$, and proton and neutron $d_{2}$ measurement. In addition to this, we will discuss the transverse spin structure of the nucleon which can be accessed using chiral-odd transversity distribution $\left(h_{1}\right)$, and show some results from measurements done on polarized ${ }^{3} \mathrm{He}$ target in Hall A.
\end{abstract}

The 8th International Workshop on Chiral Dynamics, CD2015 ***

29 June 2015 - 03 July 2015

Pisa, Italy

\footnotetext{
* Speaker.

$\dagger$ This work is supported in part through funds provided by the U.S. Department of Energy under Contract Number DE-FG02-94ER40818. The author would like to thank the spokespersons and collaborators of saGDH, EG4, SANE and E06-010(d2n) experiments at JLab for providing several useful plots and inputs.
} 


\section{Introduction}

High-energy electron scattering is a powerful techniques for studying the internal structure of nucleon. It has a long history starting from pioneering experiments done at SLAC in 60s and 70s, and later at CERN and DESY. In this process the electron scatters from a quark in the nucleon and is detected in a spectrometer. While the electron-quark interaction is described by well-known Quantum Electrodynamics (QED), the internal dynamics of the nucleon is described by Quantum Chromodynamics (QCD). QCD is well understood and verified in the perturbative region (shortdistance scale), but it is not so well understood in the non-perturbative region where quark-gluon interactions becomes very complicated to describe. Hence, at low energies, effective field theory such as Chiral Perturbation Theory $(\chi \mathrm{PT})$ is used to describe the experimental data and make predictions. The polarization of electron and nucleon provides additional degrees of freedom which can be used to construct observables to test the $\chi \mathrm{PT}$ theory. The spin of the nucleon is also an important quantity that has not been completely understood. Although, the longitudinal quark spin contribution is experimentally well measured, the contributions from transverse quark spin is still not completely understood. Several recent experiments were conducted at JLab to measured longitudinal spin structure functions $g_{1}$ and $g_{2}$ from $Q^{2}=0.02-6 \mathrm{GeV}^{2}$, and transversity $\left(h_{1}\right)$ at $Q^{2}=2.0 \mathrm{GeV}^{2}$. In this proceedings an overview of these experiments along with some of the preliminary and final results will be discussed.

\section{Inclusive Electron Scattering}

In the process of inclusive lepton-nucleon scattering a lepton $(l)$ with initial four-momentum $p^{\mu}=(E, \vec{k})$ scatters off a nucleon and is detected with final four-momentum $p^{\prime \mu}=\left(E^{\prime}, \vec{k}^{\prime}\right)$ :

$$
l(p)+N(P) \rightarrow l\left(p^{\prime}\right)+X\left(P^{\prime}\right),
$$

where the nucleon's four-momenta is $P^{\mu}=(M, \overrightarrow{0})$ since the target is at rest and the final (hadronic) undetected state is $X$. The four-momentum of exchanged virtual photon is $q^{\mu}=\left(p-p^{\prime}\right)^{\mu}=(v, \vec{q})$ with $v=E-E^{\prime}$ and the scattering angle of the lepton is given by $\theta$. The kinematics of the process can be described by following Lorentz invariant quantities:

$$
\begin{array}{r}
Q^{2}=-q^{2} \simeq 2 E E^{\prime}(1-\cos (\theta)) \\
W=\sqrt{(p+q)^{2}}=\sqrt{\left(M^{2}+2 M v-Q^{2}\right)} \\
x=\frac{Q^{2}}{2 P \cdot q}=\frac{Q^{2}}{2 M v} \\
y=\frac{q \cdot P}{p \cdot P}=\frac{v}{E},
\end{array}
$$

where $Q^{2}$ is the negative of the squared four-momentum transferred and $x$ is the Bjorken scaling variable which represents the fraction of nucleon momentum carried by the stuck quark.

In the Born approximation, the differential cross section of the inclusive electron scattering with a nucleon can be expressed as a product of leptonic tensor $\left(L_{\mu v}\right)$ and a hadronic tensor $\left(W^{\mu v}\right)$.

$$
\frac{d \sigma}{d \Omega d E^{\prime}}=\frac{\alpha^{2}}{Q^{4}} \frac{E^{\prime}}{E} L_{\mu \nu} W^{\mu v}
$$


The leptonic tensor can be calculated precisely in QED, and the hadronic tensor can be written as a combination of symmetric and antisymmetric parts. This effectively parametrizes the internal hadronic structure into spin-independent structure functions $\left(F_{1}, F_{2}\right)$ and spin-dependent structure functions $\left(g_{1}, g_{2}\right)$. In the naive parton model, $F_{1}\left(x, Q^{2}\right)$ or $F_{2}\left(x, Q^{2}\right)$ gives the quark momentum distribution and $g_{1}\left(x, Q^{2}\right)$ probes the quark longitudinal spin distribution [1].

$$
g_{1}=\frac{1}{2}\left(\frac{4}{9}(\Delta u+\Delta \bar{u})+\frac{1}{9}(\Delta d+\Delta \bar{d})+\frac{1}{9}(\Delta s+\Delta \bar{s})\right)
$$

where $\Delta u(\Delta \bar{u}), \Delta d(\Delta \bar{d})$, and $\Delta s(\Delta \bar{s})$ are the polarized quark(antiquark) distributions.

The second spin structure function $g_{2}$ has no simple interpretation in the native parton model. It is related to the quark-gluon correlation through higher twist effects. In the operator product expansion (OPE) analysis at high $\mathrm{Q}^{2}, g_{2}$ can be written as a sum of twist-2 and twist-3 terms:

$$
g_{2}\left(x, Q^{2}\right)=g_{2}^{W W}\left(x, Q^{2}\right)+\bar{g}_{2}\left(x, Q^{2}\right),
$$

where $\bar{g}_{2}$ is the higher twist (twist-3) term and $\mathrm{g}_{2}^{W W}$ is the twist-2 (Wandzura-Wilczek) term and can be determined from the twist- 2 part of $g_{1}[2]$ :

$$
g_{2}^{W W}\left(x, Q^{2}\right)=-g_{1}\left(x, Q^{2}\right)+\int_{x}^{1} \frac{g_{1}\left(y, Q^{2}\right)}{y} d y .
$$

The higher twist term $\bar{g}_{2}$ is further split into two terms - one containing leading twist transversity distribution $\left(h_{1}\right)$ suppressed by the quark mass, and a truely twist-3 part $(\zeta)$ that arises from quarkgluon interactions [3]

$$
\bar{g}_{2}=-\int_{x}^{1} \frac{d}{d y}\left[\frac{m_{q}}{M} h_{1}\left(y, Q^{2}\right)+\zeta\left(y, Q^{2}\right)\right] \frac{d y}{y} .
$$

Hence the measurement of $g_{2}$ provides a clean way to access the twist- 3 effects. Also, using the OPE, a $Q^{2}$ dependent quantity, $d_{2}$, can be constructed:

$$
d_{2}\left(Q^{2}\right)=\int_{0}^{1} x^{2}\left[2 g_{1}\left(x, Q^{2}\right)+3 g_{2}\left(x, Q^{2}\right)\right] d x .
$$

where $d_{2}$ can be related to a specific matrix element containing local operators of quarks and gluon fields, and is calculable in lattice QCD. There are two interpretations of $d_{2}$ : one where it is expressed as a linear combination of electric and magnetic "color polarizabilites", summed over quark flavors [4], and in the other it is proportional to the instantaneous average sum of the transverse electric and magnetic force the struck quark experiences at the instance it is hit by the virtual photon [5].

The spin structure functions $g_{1}$ and $g_{2}$ can be accessed experimentally by measuring the cross section differences between two different target polarization directions (longitudinal and transverse) with respect to the electron beam polarization. The cross section difference when target is longitudinally polarized $(\Uparrow)$ is given by

$$
\Delta \sigma_{\|}=\frac{d^{2} \sigma^{\downarrow \Uparrow}}{d \Omega d E^{\prime}}-\frac{d^{2} \sigma^{\uparrow \Uparrow}}{d \Omega d E^{\prime}}=\frac{4 \alpha^{2}}{M v Q^{2}} \frac{E^{\prime}}{E}\left[\left(E+E^{\prime} \cos \theta\right) g_{1}\left(x, Q^{2}\right)-2 M x g_{2}\left(x, Q^{2}\right)\right],
$$


where $\uparrow(\downarrow)$ represent the electron polarization along (opposite) to the beam direction. The cross section difference when target is transversely polarized $(\Rightarrow)$ is given by

$$
\Delta \sigma_{\perp}=\frac{d^{2} \sigma^{\downarrow \Rightarrow}}{d \Omega d E^{\prime}}-\frac{d^{2} \sigma^{\uparrow \Rightarrow}}{d \Omega d E^{\prime}}=\frac{4 \alpha^{2}}{M v Q^{2}} \frac{E^{\prime}}{E}\left[g_{1}\left(x, Q^{2}\right)+\frac{2 M}{v} g_{2}\left(x, Q^{2}\right)\right] \sin \theta .
$$

Using the two equations above one can extract both $g_{1}$ and $g_{2}$ from the measured cross sections.

\section{Moments of Spin Structure Functions and Sum Rules}

The experimentally measured spin structure functions are expressed as moments in order to compare them to theoretical calculations. For example, the $n$th moment of $g_{1}$ is given by

$$
\Gamma_{n}=\int_{0}^{1} x^{n-1} g_{1}\left(x, Q^{2}\right) d x
$$

Using the moments of structure functions several spin sum rules have been proposed which can also be used to test the theoretical predictions. Spin sum rules are important quantities that relate moments of spin structure functions to static properties of nucleon or the quantities that can be theoretically calculable. Therefore, sum rules provide an important way to study QCD.

The Ellis-Jaffe sum rule [6] relates the first moment of $g_{1}$ to the polarized quark distributions $(\Delta u, \Delta d)$ in the Bjorken limit $\left(Q^{2} \rightarrow \infty\right)$ and it was found to be violated, resulting in "spin-crisis" in 1980s. The Bjorken sum rule [7] links the isovector part of the $g_{1}$ moment to the nucleon axial charge $\left(g_{A}\right)$ that governs the neutron $\beta$-decay. It can be expressed as

$$
\Gamma_{1}^{p-n}\left(Q^{2}\right) \equiv \int_{0}^{1}\left(g_{1}^{p}-g_{1}^{n}\right)=\frac{g_{A}}{6}\left(1-\frac{\alpha_{s}}{\pi}-3.56\left(\frac{\alpha_{s}}{\pi}\right)^{2}-20.215\left(\frac{\alpha_{s}}{\pi}\right)^{3} \ldots\right)+\mathscr{O}\left(\frac{1}{Q^{2}}\right) .
$$

The Burkhardt-Cottingham (BC) sum rule [8] states that the first momentum of $g_{2}$ is zero at any $Q^{2}$ :

$$
\Gamma_{2} \equiv \int_{0}^{1} g_{2}\left(x, Q^{2}\right) d x=0 .
$$

The current results of BC sum rule for proton and neutron are shown in Fig. 1. The top plot shows the proton data and the bottom plot shows the existing neutron data for the second moment of $g_{1}$. There is not much data on proton in the low $Q^{2}$ region. Recent measurements from JLab on proton target covers this low $Q^{2}$ region.

The Gerasimov-Drell-Hearn (GDH) sum rule links the energy-weighted photoabsorption cross section, a dynamical quantity, to particle's anomalous magnetic moment $(\kappa)$, a static property in the ground state:

$$
\int_{v_{0}}^{\infty} \frac{d v}{v}\left[\sigma_{1 / 2}(v)-\sigma_{3 / 2}(v)\right]=-2 \pi^{2} \alpha \frac{\kappa^{2}}{M^{2}}
$$

where $v_{0}=m_{\pi}\left(1+\frac{m_{\pi}}{2 M}\right) \approx 150 \mathrm{MeV}$ is the threshold energy for pion production. $\sigma_{1 / 2}$ and $\sigma_{3 / 2}$ are the photoabsorption cross-sections where $1 / 2$ and $3 / 2$ are the sum of photon and target helicities in the center of mass system. The GDH integral has been generalized by replacing the photoabsorption cross sections to virtual photon cross sections [9] $\left(Q^{2}>0\right)$ where it is related to $g_{1}$ and $g_{2}$. This allows constraining GDH integral from $Q^{2}=0$ to a large value. 


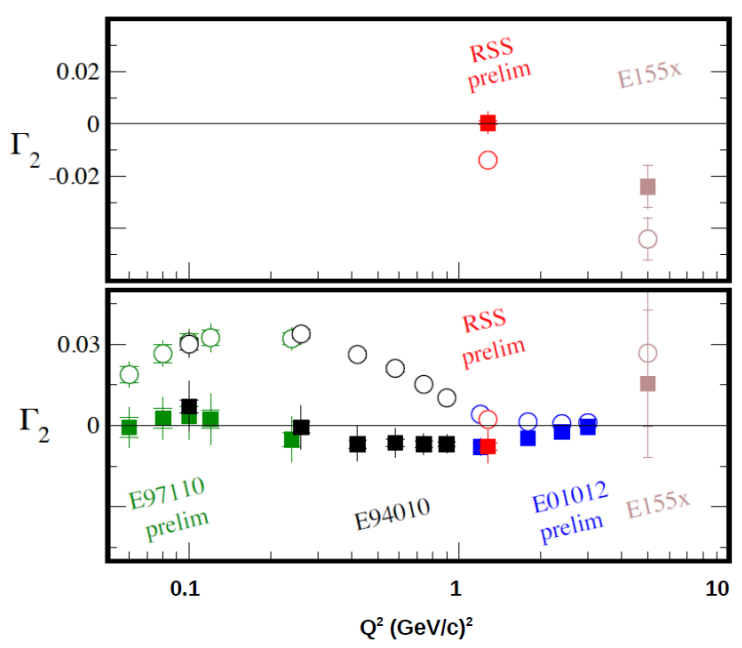

Figure 1: Current results of BC sum rule. The top plot shows proton data and the bottom plot shows neutron results. The open circles represent measured data, while black diamonds show the total integral with low- $x$ and elastic region contributions included. Reproduced from Ref. [10]

The generalized spin polarizabilities $\gamma_{0}$ and $\delta_{L T}$ describe the response of the nucleon to photon absorption and are connected to the higher moments of polarized structure functions.

$$
\begin{gathered}
\gamma_{0}\left(Q^{2}\right)=\frac{16 \alpha M^{2}}{Q^{6}} \int_{0}^{x_{0}}\left[g_{1}\left(x, Q^{2}\right)-\frac{4 M^{2}}{Q^{2}} x^{2} g_{2}\left(x, Q^{2}\right)\right] d x, \\
\delta_{L T}\left(Q^{2}\right)=\frac{16 \alpha M^{2}}{Q^{6}} \int_{0}^{x_{0}}\left[x^{2} g_{1}\left(x, Q^{2}\right)+g_{2}\left(x, Q^{2}\right)\right] d x .
\end{gathered}
$$

\section{Spin Structure Measurements at JLab}

Experiments at JLab use the polarized electron beam with energies up to $6 \mathrm{GeV}$ (now upgraded to $12 \mathrm{GeV}$ ) and polarized targets such as polarized ${ }^{3} \mathrm{He}$ or $\mathrm{NH}_{3}$ to measure the spin structure functions. The measurements discussed below were performed in the experimental halls A and B. Hall A consists of two high resolution spectrometers (HRSs) which are used to measure inclusive electron cross sections with high precision. Both polarized ${ }^{3} \mathrm{He}$ and $\mathrm{NH}_{3}$ targets have been used in Hall A for spin structure measurements. Polarized ${ }^{3} \mathrm{He}$ can be used as an effective neutron target since the ground state of ${ }^{3} \mathrm{He}$ is dominated by the $\mathrm{S}$-state, where two proton spins are anti-aligned and the remaining neutron carries most of the spin of ${ }^{3} \mathrm{He}$ nucleus. This target provides very high luminosities of up to $10^{36} \mathrm{~s}^{-1} \mathrm{~cm}^{-1}$. Polarized $\mathrm{NH}_{3}$ is used as a proton target and polarized $\mathrm{ND}_{3}$ is used as a deuteron target. These targets are polarized using Dynamic Nuclear Polarization (DNP) method, operating under very high magnetic field (5 T). Hall B measurements were made using the CLAS detector and polarized $\mathrm{NH}_{3}$ and $\mathrm{ND}_{3}$ targets. These targets provide polarized luminosities up to $10^{34} \mathrm{~s}^{-1} \mathrm{~cm}^{-1}$ using the CLAS detector.

\subsection{Longitudinal Spin Structure Measurements at Low $Q^{2}$}

The most recent JLab measurements of $g_{1}$ and $g_{2}$ at low $Q^{2}$ were performed by three experiments: E97-110 and E08-027 experiments in Hall A and the EG4 experiment in Hall B. The EG4 
experiment in Hall B [11] used CLAS detector to measure the $g_{1}$ structure function for both proton and deuteron using polarized $\mathrm{NH}_{3}$ and $\mathrm{ND}_{3}$ targets, respectively. The experiment performed doubly polarized inclusive electron scattering at low $Q^{2}\left(0.02<Q^{2}<0.7 \mathrm{GeV}^{2}\right)$. The goal of the experiment is to test the $\chi \mathrm{PT}$ as $Q^{2} \rightarrow 0$. The final analysis of the data from this experiment is under progress and very preliminary results are available for $g_{1}$ on both proton and deuteron targets.

The goal of E08-027 experiment in Hall A [12] is to extract proton $g_{2}$ structure function at low $Q^{2}$ region of $0.02<Q^{2}<0.2 \mathrm{GeV}^{2}$. The experiment measured spin-dependent cross section $\Delta_{\perp}$ and will combine with the data on $\Delta_{\|}$from EG4 experiment in Hall B to obtain $g_{2}$ for proton. The projected statistical precision and the kinematical reach for this experiment are shown in Fig. 2. The blue data points of the left side plot shows the projections for LT spins polarizibility $\delta_{L T}$ and the same for the right side plot shows the projections for second moment $\Gamma_{2}$ for proton. The experiment is currently in the advanced stages of analysis and expected to have preliminary results soon. These data will be very crucial for testing our understanding of the $\chi \mathrm{PT}$ theory and cross checking with the most recent calculations of spin polarizibilities from various theory groups [13, 14, 15].
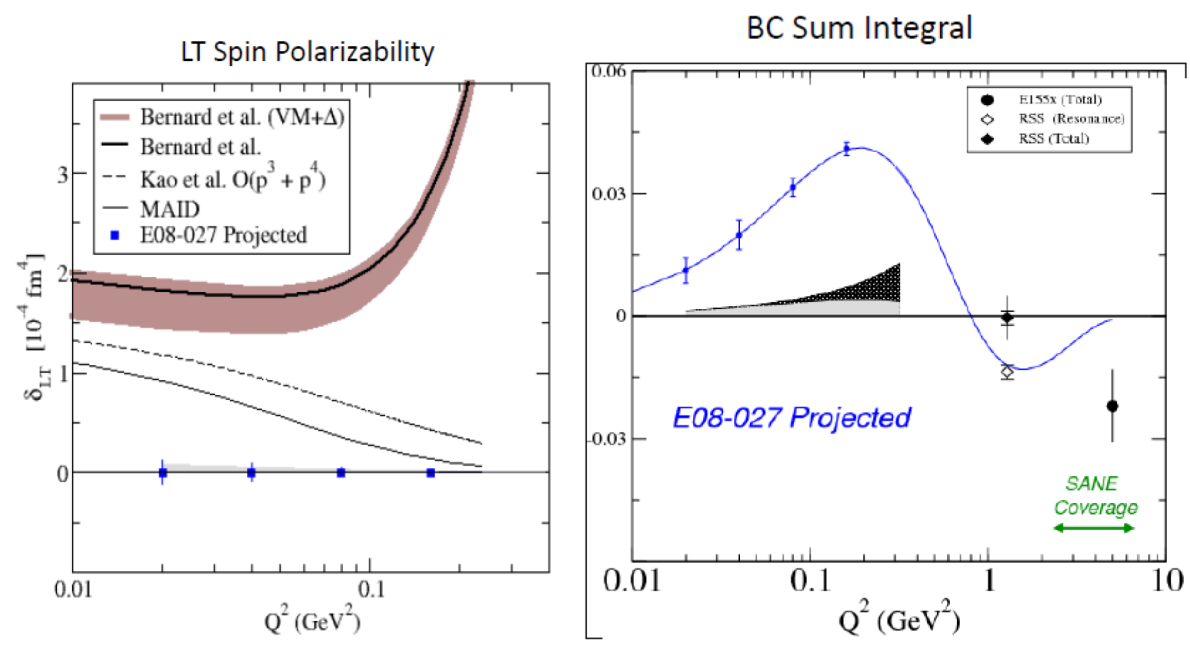

Figure 2: The projected uncertainties of $\delta_{L T}$ and $\Gamma_{1}$, along with $Q^{2}$ coverage in the E08-027 experiment.

The E97-110 experiment utilized polarized electron beam and a polarized ${ }^{3} \mathrm{He}$ target in longitudinal or in-plane transverse direction to measure doubly polarized inclusive cross section differences. Using the measured cross sections the polarized structure functions $g_{1}$ and $g_{2}$ and their moments for neutron were extracted. The goal of this experiment was to extract the GDH integral using the measured cross sections. The $Q^{2}$ range for this experiment was from $0.04-0.24$ $\mathrm{GeV}^{2}[16,17]$.

The preliminary results for first moment, $\Gamma_{1}^{n}$, are shown in Fig. 3. The results from this experiment are shown in red triangles, along with previous measurements from JLab, SLAC E143 and HERMES. The error bars include both statistical and systematic. The two model calculations shown in the plot are from $[18,19]$. The data agrees well with the models. The shaded band shows calculations with an estimate of the $\Delta$-resonance and vector meson contributions. The heavy baryon $\chi$ PT calculations [20] are shown in red dotted curve which agrees with the data only for the lower $Q^{2}$ points [17]. 


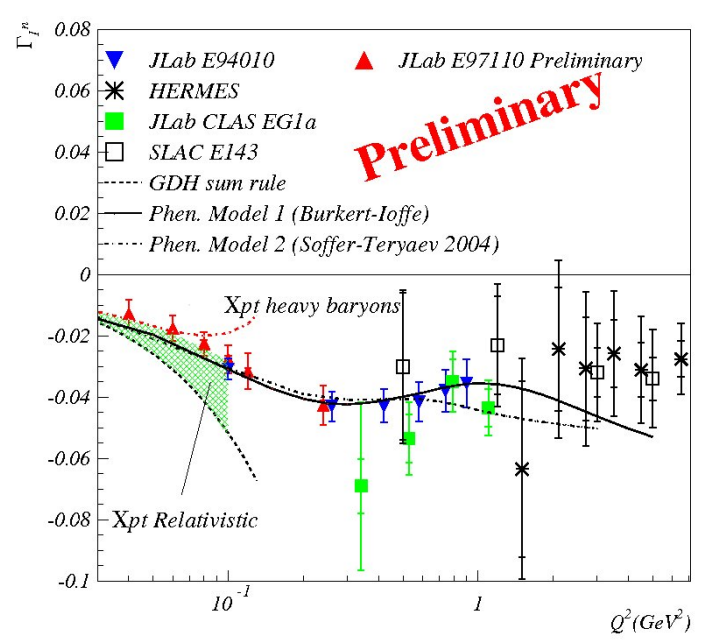

Figure 3: Preliminary results of $\Gamma_{1}$ for neutron as a function of $Q^{2}$ from E07-110 experiment are shown in red color.
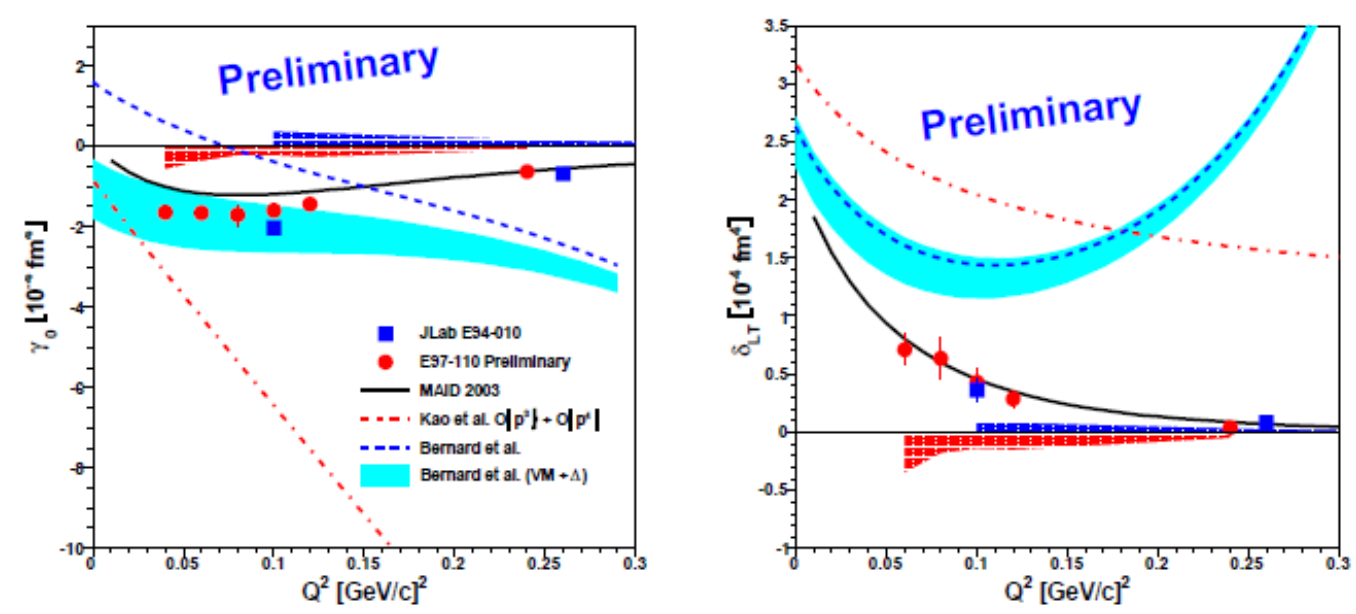

Figure 4: Preliminary results of $\gamma_{0}$ and $\delta_{L T}$ for neutron as a function of $Q^{2}$ from E07-110 experiment are shown in red color. The error bars represent statistical and the red band shows the systematics.

Using the data from this experiment the neutron spin polarizabilities $\gamma_{0}$ and $\delta_{L T}$ were extracted [16]. The preliminary results are shown in Fig. 4. The red data points show the results from this experiment, along with previous JLab measurements (shown in blue data points). The red band near zero shows the systematics uncertainties for the this experiment. For both $\gamma_{0}$ and $\delta_{L T}$ the calculations from MAID model agree reasonably well, although, at low $Q^{2}\left(<0.15 \mathrm{GeV}^{2}\right)$ the MAID model calculations are slightly higher than the data for $\gamma_{0}$. Two $\chi$ PT calculations are shown on the plot. The $\mathrm{RB} \chi \mathrm{PT}$ calculations [21] shown in light blue band agrees reasonably well with $\gamma_{0}$ data for $Q^{2}$ below $0.12 \mathrm{GeV}^{2}$. The $\mathrm{HB} \chi \mathrm{PT}$ calculation [22] shown in red dotted line has large discrepancy with the $\gamma_{0}$ data, which maybe be due to the large resonance contributions to this observable. The $\chi \mathrm{PT}$ calculations for $\delta_{L T}$ shown in the figure do not agree with the data. There are 
three recent calculations of $\delta_{L T}$ using $\mathrm{B} \chi \mathrm{PT}$ approach [13], covariant $\mathrm{B} \chi \mathrm{PT}$ [14] and using axial anomaly contribution [15]. The $\mathrm{B} \chi \mathrm{PT}$ calculations by V. Lensky et al., for the neutron $\delta_{L T}$ shows reasonable agreement with MAID model estimations and the experimental data.

\subsection{High $Q^{2}$ Measurements of Longitudinal Spin Structure Function}

Most recent high $Q^{2}$ measurements of $g_{1}$ and $g_{2}$ at JLab were performed by two experiments: the Spin Asymmetries of the Nucleon Experiment(SANE) in Hall C [23] and E06-014 (d2n) experiment in Hall A [24]. The goal of the SANE experiment was to measure the proton $g_{2}$ and spin asymmetry $A_{1}$ in the kinematic range $2.5 \leq Q^{2} \leq 6.5 \mathrm{GeV}^{2}$ and $0.3 \leq x \leq 0.8$. The experiment used polarized electron beam of $4.7 \mathrm{GeV}$ and $5.9 \mathrm{GeV}$ on a polarized ammonia $\left(\mathrm{NH}_{3}\right)$ target to measure the spin asymmetries, $\mathrm{A}_{180}$ and $\mathrm{A}_{80}$, where 180 and 80 indicates the target polarization anti-parallel and near-perpendicular to the incoming beam direction. The experiment used a large acceptance electron detector which consists of a forward tracker, gas Cherenkov detector, a lucite hodoscope and an electromagnetic calorimeter. The preliminary results of proton $g_{1}$ and $g_{2}$ from SANE experiment are shown in Fig. 5. The data from different $Q^{2}$ is shown in color, along with the previous world data which is shown in black. The left side plot shows $x^{2} g_{1}$ for proton as a function of $x$ and the right side plot shows $x^{2} g_{2}$ for proton. The systematic bands are shown below the data points with corresponding color. This experiment extended the kinematic range from low $x$ region to high $x$ region. These data will be used to extract the proton twist- 3 matrix element $d_{2}$.

The E06-014 experiment measured the double spin asymmetries (DSA) in deep inelastic and resonance region at large $x(0.2 \leq x \leq 0.9)$ using $4.7 \mathrm{GeV}$ and $5.9 \mathrm{GeV}$ polarized beam and a polarized ${ }^{3} \mathrm{He}$ target in longitudinal and transverse direction [25]. The data was used to extract $g_{1}$ and $g_{2}$ from ${ }^{3} \mathrm{He}$ target which in turn was used for the extraction of neutron twist-3 matrix element $d_{2}^{n}$ at $\left\langle Q^{2}>\right.$ of $3.21 \mathrm{GeV}^{2}$ and $4.32 \mathrm{GeV}^{2}$. The experiment used two spectrometers, a high resolution spectrometer (HRS) for the cross section measurements, and a large acceptance BigBite spectrometer for asymmetry measurements in Hall A.
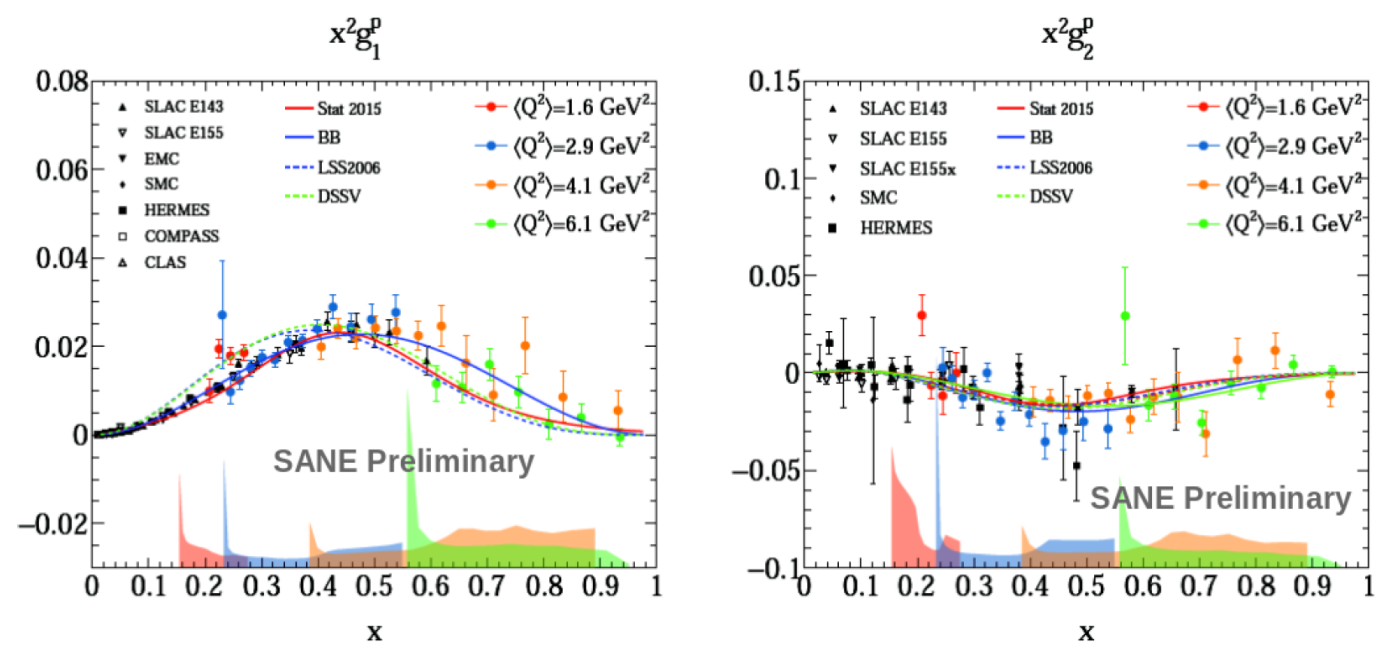

Figure 5: Preliminary results of $x^{2}$-weighted $g_{1}$ (left) and $g_{2}$ (right) for proton as a function of $x$ from SANE experiment. The colored data are from SANE experiment at different $Q^{2}$. The data from past experiments are also shown in black color. 


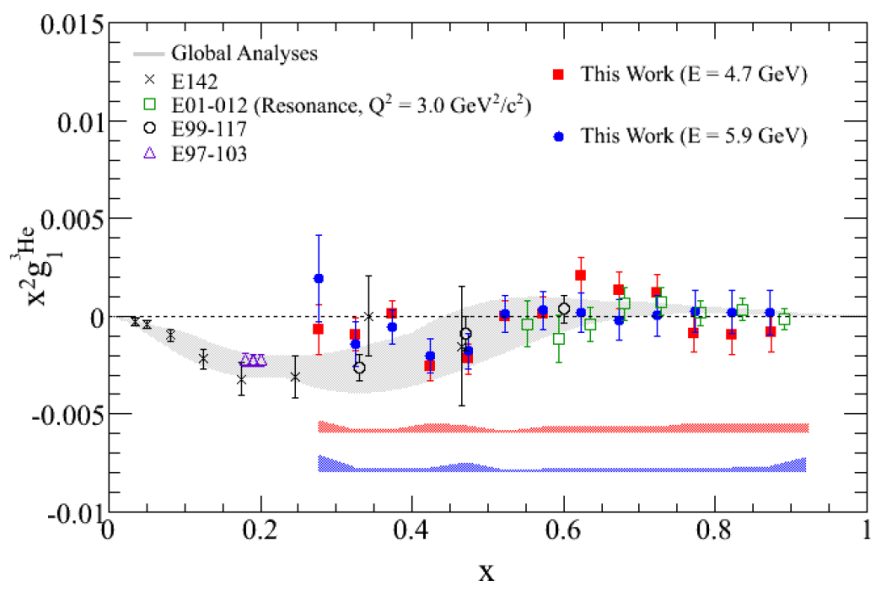

Figure 6: $x^{2}$-weighted $g_{1}$ for ${ }^{3} \mathrm{He}$ plotted as a function of $x$. The red and blue data points are from E06-014 experiment. The blue and red bands on the plot represents systematics and the error bar represent statistical.

The $x^{2}$-weighted polarized spin structure function $g_{1}$ of ${ }^{3} \mathrm{He}$ is shown in Fig. 6. The data is consistent with the previous experiments at JLab and SLAC. The $x^{2}$-weighted polarized spin structure function $g_{2}$ of ${ }^{3} \mathrm{He}$ is shown in Fig. 7. These data improved the precision of $g_{2}$ dramatically over the previous measurements from JLab and SLAC. The bottom plot of Fig. 7 shows the same results as the top but with y-axis zoomed by a factor 10. The measured DSAs and cross sections at each beam energy were used to extract $d_{2}$ for ${ }^{3} \mathrm{He}$. The neutron information was extracted from ${ }^{3} \mathrm{He}$ using effective polarization approach [25].

\subsection{Transverse Spin Structure Measurements}

Transversity distribution $\left(h_{1}\right)$ is the third important parton distribution, along with unpolarized distribution $\left(f_{1}\right)$ and longitudinal spin distribution $\left(g_{1}\right)$, that describes the nucleon structure in leading order. Transversity describes the distribution of transversely polarized quarks in a transversely polarized nucleon. This can be studied in semi-inclusive deep inelastic scattering (SIDIS) where a leading hadron is detected in coincidence with the scattered electron. Another interesting distribution that can be accessed in SIDIS process is the Sivers function. The Sivers function provides the number density of unpolarized partons inside a transversely polarized proton, and it requires wave function components with nonzero orbital angular momentum and thus provides information about the correlation between the quark orbital angular momentum (OAM) and nucleon spin.

The E06-010 experiment at JLab measured the Collins and Sivers moments on effective neutron target which will be used in extracting the transversity and Sivers distribution functions, respectively [26]. The measurement was done by scattering off longitudinally polarized electrons on a transversely polarized ${ }^{3} \mathrm{He}$ target in the SIDIS reaction ${ }^{3} \mathrm{He}\left(e, e^{\prime} h\right) \mathrm{X}$, where $h$ is a charged hadron $\left(\pi^{ \pm}\right.$or $\left.K^{ \pm}\right)$. An electron beam with energy of $5.9 \mathrm{GeV}$ was used. The scattered electrons were detected in the large acceptance BigBite Spectrometer and the produced hadrons were detected in the standard Hall A spectrometer. The target spin was flipped every 20 minutes and the single spin asymmetry was constructed from the data collected in each spin state. The kinematics were focused on the valence quark region. 


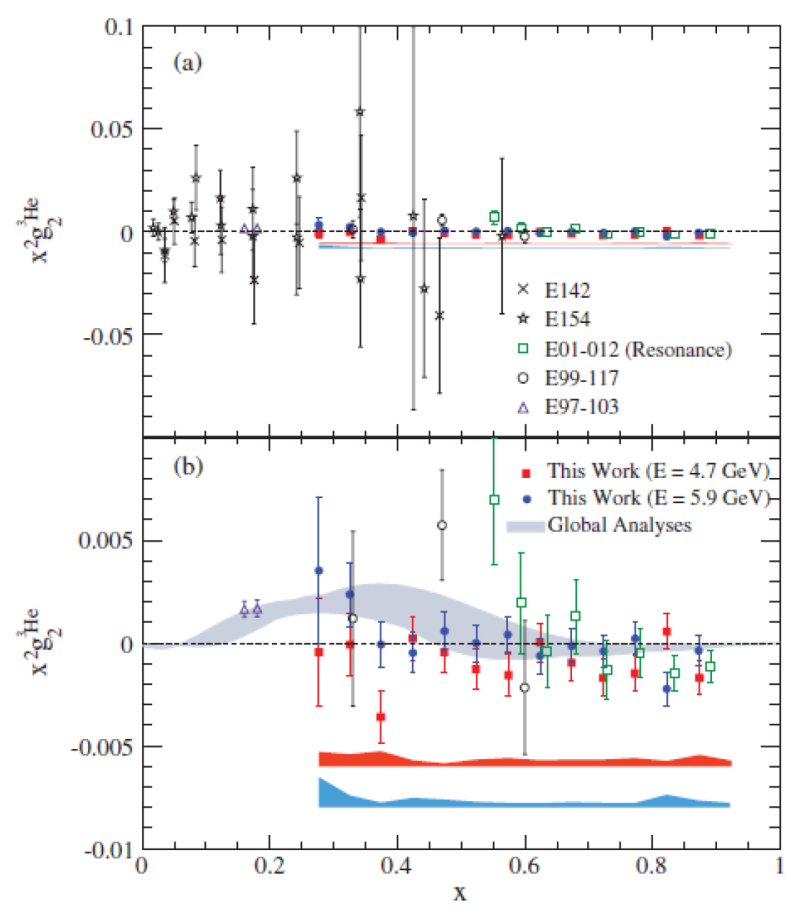

Figure 7: $x^{2}$-weighted $g_{2}$ for ${ }^{3} \mathrm{He}$ plotted as a function of $x$. The world data is shown in black and the data from E06-014 is shown in color. The blue and red bands on the bottom plot (b) represents systematics and the error bar represent statistical. The bottom plot is a zoomed by a factor 10 from the top plot.

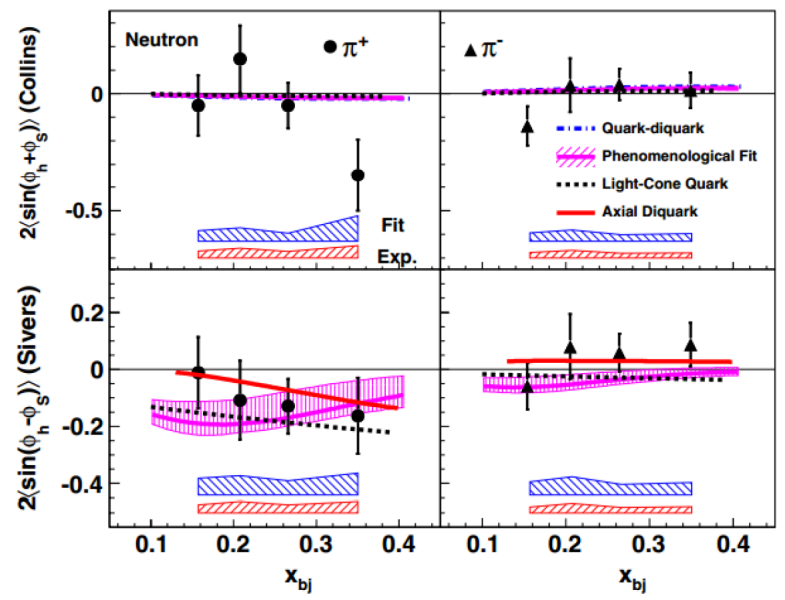

Figure 8: The extracted neutron Collins (top) and Sivers (bottom) moments vs $x$ for the electroproduction of $\pi^{+}$(left) and $\pi^{-}$(right) from ${ }^{3} \mathrm{He}$ target. The bands below the data points represent the experimental and model uncertainties, which are labeled as Exp and Fit, respectively. 
The results for the extracted neutron Collins and Sivers moments from the ${ }^{3} \mathrm{He}$ asymmetries are shown in Fig. 8 in the top and bottom panels, respectively. The left-hand side of the plot shows the results for the $\pi^{+}$and the right-hand side shows the results for $\pi^{-}$. The error bars on the points indicate the statistical precision of the data, whereas the bands on the bottom show the experimental (Exp) and fitting (Fit) systematic uncertainties. The total experimental uncertainties are less than $25 \%$ of the statistical uncertainty in each bin. The fitting uncertainties result from ignoring the pretzelosity and higher twist terms in the extraction of the moments. The data are compared with a phenomenological fit [27] and model calculations, which include a light-cone constituent quark model (LCCQM) [28, 29] and quark-diquark calculations [30, 31]. Both the data and the calculations indicate that the Collins asymmetries are small, though the data at $x=0.34$ is more negative at the $2 \sigma$ level. For the Sivers moments, the $\pi^{+}$asymmetries favor negative values, whereas the $\pi^{-}$results are consistent with zero within the uncertainties. These data, along with the data on proton target from HERMES and COMPASS experiments were used in a recent global analysis for extracting transversity distribution [32].

\section{Summary}

We discussed recent results of nucleon structure functions $g_{1}$ and $g_{2}$ from the measurements performed at JLab. The low $Q^{2}$ measurements of spin polarizabilites, $\gamma_{0}$ and $\delta_{L T}$, provide crucial data to check the $\chi \mathrm{PT}$ calculations as $Q^{2} \rightarrow 0$. The high $Q^{2}$ data of $g_{1}$ and $g_{2}$ were measured on both proton and neutron targets. Using these data the twist- 3 matrix element, $d_{2}$, is extracted which will provide test of lattice QCD predictions. Finally, the transverse spin structure was studied at JLab by measuring Collins and Sivers moments on an effective neutron target for the first time. These data are used in the global analysis for the extraction of transversity distribution function.

\section{References}

[1] S. E. Kuhn, J. P. Chen and E. Leader, Prog. Part. Nucl. Phys. 63, 1 (2009).

[2] S. Wandzura and F. Wilczek, Phys. Lett. B 72, 195 (1977).

[3] J.L. Cortes, B. Pire, J.P. Ralston, Z. Phys. C - Particles and Fields 55, 409-416 (1992).

[4] X.-D. Ji, Proceedings of the 7th International Conference on the Structure of Baryons, Santa Fe, 1995, arXiv:hep-ph/9510362.

[5] M. Burkardt, Phys. Rev. D 88, 114502 (2013).

[6] J. Ellis and R. L. Jaffe, Phys. Rev. D 9, 1444 (1974).

[7] J. D. Bjorken, Phys. Rev. 148, 1467 (1966).

[8] H. Burkhardt and W. N. Cottingham, Ann. Phys. (N.Y.) 56, 453 (1970).

[9] M. Anselmino, B. L. Ioffe and E. Leader, Sov. J. Nucl. Phys. 49, 136 (1989).

[10] J.-P. Chen, Int. J. Mod. Phys. E, 19, 1893 (2010).

[11] M. Battaglieri, A. Deur, R. De Vita and M. Ripani, JLab experiment E03-006.

[12] A. Camsonne, J. P. Chen and K. Slifer, JLab experiment E08-027. 
[13] V. Lensky, J. M. Alarcon and V. Pascalutsa, Phys. Rev. C 90, 055202 (2014).

[14] V. Bernard, E. Epelbaum, H. Krebs, Ulf-G. Meißner, Phys. Rev. D 87, 054032 (2013).

[15] N. Kochelev and Y. Oh, Phys. Rev. D 85, 016012 (2012).

[16] V. Sulkosky, The Spin Structure of ${ }^{3} \mathrm{He}$ and the Neutron at Low $Q^{2}$ : A Measurement of the Generalized GDH Integrand, http://hallaweb.jlab.org/experiment/E97-110/thesis.html.

[17] V. Sulkosky, An Overview of Longitudinal Spin Structure Measurements from JLab, Proceedings of Science, PoS(CD12)023, Chiral Dynamics 2012.

[18] J. Soffer and O. V. Teryaev, Phys. Rev. D 70, 116004 (2004).

[19] V. D. Burkert and B. L. Ioffe, Phys. Lett. B 296, 223 (1992).

[20] X. Ji, C. Kao and J. Osborne, Phys. Lett. B 472, 1 (2000).

[21] V. Bernard, T. R. Hemmert and U.-G. Meißner, Phys. Lett. B 545, 105 (2002); Phys. Rev. D 67, 076008 (2003).

[22] C. W. Kao, T. Spitzenberg and M. Vanderhaeghen, Phys. Rev. D 67, 016001 (2003).

[23] S. Choi, M. Jones, Z-E. Meziani and O. A. Rondon, JLab experiment E07-003 (SANE).

[24] B. Sawatzky, S. Choi, X. Jiang and Z.-E.Meziani, JLab experiment E06-014 (d2n).

[25] M. Posik et al., Phys. Rev. Lett. 113, 022002 (2014).

[26] X. Qian. et al. Phys. Rev. Lett. 107, 072003 (2011).

[27] M. Anselmino et al. Phys. Rev. D 75, 054032 (2007).

[28] S. Boffi et al. Phys. Rev. D 79, 094012 (2009).

[29] B. Pasquini, S. Cazzaniga, and S. Boffi. Phys. Rev. D 78, 034025 (2008).

[30] J. She and B. Q. Ma. Phys. Rev. D 83, 037502 (2011).

[31] B. Q. Ma, I. Schmidt, and J. J. Yang. Phys. Rev. D 65, 034010 (2002).

[32] Z-B Kang, A. Prokudin, P. Sun, F. Yuan, arXiv:1505.05589. 